\title{
Cognitive impairment in 873 patients with idiopathic Parkinson's disease
}

\section{Results from the German Study on Epidemiology of Parkinson's Disease with Dementia (GEPAD)}

\author{
Oliver Riedel, Jens Klotsche, Annika Spottke, Günther Deuschl, Hans Förstl, Fritz Henn, \\ Isabella Heuser, Wolfgang Oertel, Heinz Reichmann, Peter Riederer, Claudia Trenkwalder, \\ Richard Dodel, Hans-Ulrich Wittchen
}

\begin{abstract}
Background Parkinson's disease (PD) is often accompanied by non-motor complications, such as dementia, depression, and psychotic symptoms, which worsen the prognosis and increase the personal and socioeconomic burden of disease. Prevalence estimates of these complications are quite variable and are lacking for the outpatient care sector.

Methods As part of a larger, nationwide, cross-sectional epidemiological study in $\mathrm{n}=315$ neurological outpatient settings in Germany, this paper estimates the frequency of dementia and cognitive impairment in $n=873$ outpatients meeting the UK Brain Bank criteria for idiopathic PD. Assessments were based on a clinical interview and neuropsychological assessments, including the Hoehn \& Yahr rating and Unified Parkinson's Disease Rating Scale (UPDRS). Cognitive impairment was assessed by the Mini-Mental State Exam (MMSE), Clock Drawing Test (CDT) and the Parkinson

Neuropsychometric Dementia Assessment (PANDA) and the clinician's diagnosis of dementia was based on the diagnostic criteria of DSMIV. Results Using standardized cutoff scores, the prevalence of cognitive impairment in the study sample as measured by various methods was $17.5 \%$ by MMSE ( $\leq$ $24), 41.8 \%$ by CDT $(\geq 3), 43.6 \%$ by PANDA $(\leq 14)$, and $28.6 \%$ met the DSM-IV criteria for dementia. All estimates increased with age and PD severity. Gender was an inconsistent contributor while illness duration had no significant impact on cognition. Multiple regression analyses revealed PD severity to be the strongest predictor of dementia risk (OR=4.3; $95 \% \mathrm{CI}: 2.1-9.1$ ), while neuropsychiatric syndromes had independent, although modest additional contributions $(\mathrm{OR}=2.5,95 \%$ CI: $1.6-3.8)$.

Conclusion Estimates of cognitive impairment and dementia in PD patients are largely dependent on the diagnostic measure used. Using established clinical diagnostic standards for dementia the overall rate on routine outpatient neurological care is $28.6 \%$, but using more sensitive neuropsychological measures, rates for cognitive impairment might be up to 2-fold higher. The MMSE revealed strikingly low sensitivity. Neuropsychiatric syndromes, in addition to PD severity and age, have an independent - although modest - additional contribution to patients' risk for cognitive impairment and dementia.
\end{abstract}

Key words Parkinson's disease, dementia, cognitive impairment, MMSE, CDT, PANDA

\section{Introduction}

Parkinson's disease (PD) is one of the most common neurodegenerative disorders of late life, with an estimated prevalence of 2-3\% among people 65 years old and over, and as high as $10 \%$ among those 80 years old and over [6]. Although PD is primarily characterized by resting tremor, bradykinesia, rigidity, and postural instability, non-motor symptoms such as cognitive impairment, dementia and depression may contribute to a more malignant course of illness, increase the risk of nursing home placement, and exacerbate personal and socioeconomic burden $[4,16,19]$. Cognitive impairment and dementia (Parkinson's Disease dementia, PDD) have been highlighted as particularly common non-motor complications. There is considerable variation in the reported prevalence of PDD, making it difficult to accurately estimate its specific age, gender and illness duration- related characteristics. For 
example, the estimated prevalence of cognitive impairment in cross-sectional investigations of PDD ranges from 20-44\%, constituting an approximate 3- to 6-fold increased risk for PD patients as compared to non-PD patients $[2,12]$. The cumulative incidence of dementia reported in a longitudinal study by Aarsland et al. was 52\% after 4 years and nearly $80 \%$ after 8 years [1]. Variations in findings are most likely due to differences in study population (e.g. specialized clinics versus inpatient facilities), sampling procedures (e.g. age range), and sample size, as well as use of different diagnostic instruments, criteria and designs. Thus, the prevalence of cognitive impairment and dementia in PD at different stages and ages remains unclear. Similarly the report of other clinically relevant symptoms, such as depression and psychosis, and their impact is incomplete, as only a few studies have comprehensively examined the associations between these various non-motor complications and PD. Another noteworthy deficit is a lack of estimates derived from neurological outpatient settings in routine care. Such data are of interest for improved care and a more adequate provision of treatment resources.

With this background, the German Study on Epidemiology of Parkinson's Disease with Dementia (GEPAD) was launched to provide more comprehensive data on the epidemiology of non-motor symptoms among patients with PD treated in the neurological outpatient sector. Particular focus was placed on the estimation of the prevalence of dementia and cognitive impairment (by various methods), the assessment of depressive and psychotic symptoms, and the impact of biosocial and clinical variables on the likelihood of cognitive decline in PD patients.

\section{Methods}

Design and sampling procedure The GEPAD is a large-scale, nationwide, cross-sectional, epidemiological study of the frequency of cognitive impairment, dementia, and depressive and psychotic symptoms in an unselected sample of $n=1,749$ outpatients with parkinsonian syndromes. Patients were recruited from a national representative sample of $n=315$ officebased neurologists in Germany (Fig. 1). In order to obtain an unbiased selection of PD patients, physicians were first requested in a pilot study [28] to enroll all their PD patients on one pre-selected day. For the main study that took place on a pre-determined study day during the period of September-October 2005, at least five randomly chosen attending patients with parkinsonian syndromes were approached and recruited. The ethics committee of the Medical Faculty of the TU Dresden (No. EK140082005) approved the study. Participating patients or their caregivers gave written informed consent to be assessed according to the study protocol. The study center was blinded to patient identity.

Patients of all ages with the clinician's diagnosis of Parkinsonian syndromes were eligible. Exclusion criteria were minimal to reflect as much as possible the situation in routine care. Thirty-nine patients (2.2\% of total sample) were excluded because of ethical reasons, because of the acute severity of the patients' health condition (i.e. referral to hospital), which prohibited the administration of the assessment interview. One-hundred thirty-nine $(7.9 \%)$ were excluded, because the physicians dealt with other more acute medical problems (e.g. acute patient emergencies) of the patient on the study day. Eighty-nine patients (5.1\%) did not give written informed consent for participation and $n=33$ patients $(1.9 \%)$ were excluded for other reasons (e.g. language issues, patients with severe deficits in seeing or hearing). The final response rate among all eligible patients with parkinsonism was $82.3 \%(n=1,449)$. For the purpose of this paper, we limited the analyses to the more diagnostically homogenous group of patients meeting the UK Brain Bank criteria for idiopathic PD [14]. We used a standardized diagnostic appraisal for all patients which was bradykinesia and at least 1 of 3 additional cardinal signs (resting tremor, rigidity, and postural instability). Diagnosis of 
idiopathic PD additionally required the presence of at least 3 of the following signs: asymmetric onset, resting tremor, substantial levodopa response, and disease duration $\geq 10$ years. Patients with cerebellar signs, early severe dementia with disturbances of memory, language and praxis or other symptoms precluding the diagnosis of idiopathic Parkinson were excluded. In total, $n=873$ out of the $n=1,449$ patients met these study criteria.

Study instruments After being trained and supported by monitors, participating physicians examined all patients using a standardized interview and an assessment battery. Only established robust assessment tools that are used in physicians' daily practice were utilized. The battery contained 3 sections. Section A documented patient demographics such as age, gender, marital status, and living situation. Section B documented PD diagnostic status, incorporating the Clinical Global Impression scale (CGI) of PD severity, the Hoehn \& Yahr scale (HY) [13], and parts I (Mentation, Behavior, and Mood), II (Activities of Daily Living), and IV (Complications) of the Unified Parkinson's Disease Rating Scale (UPDRS) [10], and recording information about age of PD onset, presence of neurological symptoms, and concomitant disorders. Section C consisted of scales and ratings for neuropsychiatric complications.

Cognitive status was assessed with the Mini Mental State Exam (MMSE) and the ClockDrawing Test (CDT) $[11,26]$. Any score $\leq 24$ points in MMSE (30 maximum) or $\geq 3$ in CDT ( 6 maximum with: $1=$ best, $6=$ worst performance) was considered an appropriate cutoff for determination of cognitive impairment [32]. A randomly selected subsample of $n=289$ patients was additionally tested with the Parkinson Neuropsychometric Dementia Assessment (PANDA), a new psychometric tool with high sensitivity. The structure and the psychometric properties of the PANDA instrument as well as the underlying theory have been described in detail in recent publications $[17,18]$. In short, the PANDA assesses functions that are typically affected in PD on five subscales (word pair associate learning with immediate (task one) and delayed recall (task five), alternating verbal fluency task (task two), visuospatial task (task three) and working memory and attention task (task four) - for a maximum score of 30 points. If test results are $\geq 18$ points, this reflects a normal cognitive function level, 15-17 points suggest "mild cognitive dysfunctions" and any score below 15 indicates "severe cognitive impairment", indicative of dementia.

The clinical diagnosis of dementia was based on the DSM-IV criteria [5] as assessed in the clinical interview in part A. Severity for both, dementia and PD were rated by the physician using the 7-point Clinical Global Impression (CGI) scale [25]. Depression was screened with the Montgomery-Asberg Depression Rating Scale (MADRS); patients with a score $\geq 14$ (60 maximum) were classified as depressed [20, 22, 23]. Presence of any additional psychotic symptoms was rated by the physician on a symptom list.

The sequence of administration of the evaluation tools was the same for all patients. Quality assurance was checked by random quality assessment by study monitors in a random subset of $12 \%$ of all assessments. Data quality was checked in the study center, all patient records were checked for completeness and consistency before entry. All clock and pentagon drawings (i.e. the "copy" task in the MMSE) were independently rated by two blinded experienced clinicians. In case of discrepancy between their and the physicians' ratings (8\%) a consensus rating was used.

Data analysis Primary outcome variables were the degree of cognitive impairment based on the MMSE, CDT and PANDA score, and DSM-IV-based diagnosis. Associations between these outcomes and categorical predictor variables were investigated by logistic regression analyses. Odds ratios (ORs) and 95\% confidence intervals (CIs) are reported. The CIs for the prevalence estimates were calculated with the biascorrected and accelerated bootstrap method [8]. Multivariate regression analyses were used to investigate in a stepwise manner the association between dementia (DSM-IV) and depression, psychotic symptoms, and PD severity. Statistical inferences were based on a 5\% significance level. Because patients were 
clustered in office-based neurological settings, observations within a setting were correlated. To account for the stratified sampling, standard errors, CIs, and p-values were calculated using the Huber-White sandwich estimator [29]. All statistical analyses were performed with software package STATA (Version 9.2, Stata Corporation, College Station, TX, USA).

\section{Results}

Characteristics of the study sample The patients were primarily male (62.1\%), and their mean age was 70.5 years. The average PD onset was 63.8 years (Table 1). According to the HY scale, $12.3 \%$ of patients were stage I, $27.6 \%$ were stage II, $40.4 \%$ were stage III, and $19.8 \%$ were past stage III. Most patients were capable of running a household on their own $(23.3 \%)$ or with their spouses $(70.4 \%)$ (data not shown).

Cognitive impairment and dementia In total, $28.6 \%$ (95\% CI: 25.5-31.7) of patients met criteria for dementia according to DSM-IV criteria. Rates of cognitive impairment differed markedly by diagnostic measure used. As shown in rates by age group and gender in Fig.2A$\mathrm{D}$, impairment rated by MMSE scores $(\leq 24)$ were consistently the lowest (overall $17.5 \%$, 95\% CI: 14.9-20.2), whereas all other methods resulted in substantially higher rates. Using the CDT (score $\geq 3$ ), $41.8 \%$ (95\% CI: 38.6-45\%) of patients were determined to have cognitive impairment, compared to $43.6 \%$ (95\% CI: 37.8-49.4) using the PANDA (score $\leq$ 14). The frequency of dementia and cognitive impairment increased significantly with age with all four measures. Except for the CDT where women scored significantly worse than men, there were no gender differences. For patients aged $\geq 76$ years, the probability of a score $\geq 3$ was 2-fold higher for females versus males ( $\mathrm{OR}=2.0,95 \% \mathrm{CI}$ : $1.2-3.5$ ).

Table 2 reports the association of these cognitive impairment measures and dementia according to DSM-IV respectively with selected sociodemographic predictors. All measures were significantly associated with age, lowest rates were found for the youngest (range:8.8 MMSE to $32.8 \%$ PANDA) and highest in the oldest age group (range: 26\% MMSE to $51.1 \%$ PANDA). Patients aged $\geq 76$ years were 4 -fold more likely to have dementia than patients aged $\leq 65$ years $(\mathrm{OR}=4.0,95 \% \mathrm{CI} 2.6-6.3)$. Except for the CDT no association with gender was found. In each measure, higher educational status was associated with decreased rate of cognitive impairment, however, for the PANDA this association was not significant.

All four measures were also significantly associated with PD severity (Hoehn \& Yahr rating). Dementia according to DSM-IV occurred in $10.6 \%$ of all patients staged I, in $20.5 \%$ of those staged II, $30.6 \%$ at stage III and $48.2 \%$ in patients past stage III (i.e. IV+V, see Table 3 ). For the MMSE, there was a steady increase across stages in the percentage of patients achieving a score indicative of cognitive impairment ( $\leq 24$ points), increasing from $7.9 \%$ for stage I to $33.1 \%$ for stages IV/V. In comparison, the frequency of cognitive impairment by CDT (score $\geq 3$ ) and PANDA (score $\leq 14$ ) was higher across stages, increasing from $30.4 \%$ for stage I to $53.1 \%$ for stage IV/V (PANDA: from $18 \%$ for stage I to $62 \%$ for stage IV/V). Again, the gender difference in performance of the CDT was apparent across stages and significant for stages II (51.7 \% females vs. $22.7 \%$ males) and III (53.7 \% females vs. $41.4 \%$ males).

Using the DSM-IV criteria as the gold standard, we compared sensitivity and specificity for the three measures of cognitive impairment. Sensitivity was lowest for the MMSE (50\%) and highest for the PANDA (73.2 \%). The sensitivity of the CDT was $71.1 \%$. The specificities for the MMSE, CDT and PANDA were $94.5 \%, 69.3 \%$ and $71.4 \%$, respectively. In the MMSE and CDT, sensitivity was lowest in patients $\leq 65$ years $(32.3 \%$ and $38.7 \%)$ and highest in patients aged $71-75$ years (MMSE: $60.5 \%$ ) and $\geq 76$ years (CDT: $82.8 \%$ ). For PANDA the sensitivity rates were $75 \%$ ( $\leq 65$ years), $61.3 \%$ (66-70 years), $88.9 \%$ ( $71-75$ years) and $75 \%$ ( $\geq 76$ years). On all three measures, specificity decreased with increasing age. 
Since it has been assumed that measurements of cognitive impairment by test with motor components could be influenced by the motor dysfunction of PD, we also investigated whether the association of PD severity with cognitive impairment is influenced in this regard. We therefore used two regression analyses with each MMSE score and CDT score as a dependent dimensional variable and the PD severity as independent variable with HY stage I as reference. For the MMSE the mean total score loss across HY stages II, III and IV/V were $\Delta=-0.2, \Delta=-1.0$ and $\Delta=-2.8$, respectively. When controlling for motor dysfunction (UPDRS item "handwriting"), the mean score differences across these stages were $\Delta=-0.0, \Delta=-0.8$ and $\Delta=-2.4$. Similar results were obtained for the CDT with mean score increments of $\Delta=0.1$, $\Delta=0.3$ and $\Delta=0.8$ prior to and $\Delta=0.0, \Delta=0.3$ and $\Delta=0.6$ after adjusting for motor complications. Overall, these results suggest that the consideration of motor dysfunction attenuates the performance in the tests only marginally.

Frequency and interactions with other neuropsychiatric symptoms Overall, 26.2\% of subjects exceeded the MADRS depression cut-off score. Women were significantly more likely to be depressed (males $21.9 \%$, females $33.1 \%$; OR=1.8, 95\% CI 1.3-2.4). Nineteen percent of patients were determined to have at least one psychotic symptom; there was no significant gender difference (males $20.1 \%$, females $17.2 \%$ ). Hallucinations were the only symptom to increase significantly with age, with highest rates in patients aged $\geq 76$ years $(16.9 \%)$. Table 3 shows the number, percentage, and crude ageadjusted associations of selected predictor PD and neuropsychiatric variables with dementia and the three measures of cognitive impairment, respectively. As mentioned previously, for all four measures, the frequency of dementia and cognitive impairment was strongly associated with PD severity. Age of PD onset and PD duration were not associated with a higher probability of dementia or cognitive impairment, except for the PANDA, which was significantly inversely associated with age of PD onset (OR=0.3; 95\% CI: 0.1-0.8). Dementia and cognitive impairment were also strongly associated with depression according to MADRS (for dementia OR=3.7, 95\% CI 2.6-5.2) and psychotic symptoms (OR=4.1, 95\% CI 2.8-6.0). It is noteworthy that the association between PANDA and depression as well as psychotic symptoms was somewhat weaker compared to all measures.

Based on these significant associations, we examined the combined effect of depressive and psychotic symptoms and PD severity on the risk of dementia, taking age into account. A multiple stepwise logistic regression model was used, with only age entered initially (OR=1.06, 95\% CI: 1.04-1.08, R2=0.042). Addition of HY stage significantly increased the predictive value ( $\mathrm{R} 2=0.086$, highest for stage IV/V with an $\mathrm{OR}=6.26,95 \%$ CI: 3.11-12.62). To determine which of the variables (age, PD severity (HY) or disease duration) had the greatest impact on risk of cognitive impairment, we entered these variables in a separate logistic regression model. Our results indicate that severity of PD has a higher impact on the probability of being diagnosed with dementia $(\mathrm{OR}=2.13,95 \% \mathrm{CI}$ : $1.68-2.70)$ than did age $(\mathrm{OR}=1.06,95 \% \mathrm{CI}: 1.03-1.08)$, whereas the effect of disease duration remained insignificant (OR=1.01, 95\% CI: 0.98-1.04). Depressive symptoms determined by the MADRS $(\mathrm{R} 2=0.123)$ and presence of any psychotic symptom $(\mathrm{R} 2=0.142)$ were entered in steps three and four. The inclusion of neuropsychiatric symptoms slightly attenuated the effect of PD severity, thus improving the prediction of dementia. We also examined whether the number of psychotic symptoms markedly improved the prediction accuracy, and found no effect $(\mathrm{R} 2=0.145)$. Thus, approximately the same amount of variance was explained by the addition of any number of psychotic symptoms. 


\section{Discussion}

We investigated the frequency of cognitive impairment, clinically diagnosed dementia and other neuropsychiatric disorders (depression and psychotic symptoms) in a large unselected nationwide sample of 873 PD patients visiting office-based neurologists in Germany. Overall, the findings highlight the considerable burden of nonmotor complication in patients with PD. Strengths of the study include the large sample size, the representative sample for the outpatient sector, and the use of various established measures for the assessment of cognitive impairment and neuropsychiatric symptoms, allowing a broader and more comprehensive epidemiological characterization. The limitations are the cross-sectional design, and due to the study being conducted in routine care, the partial lack of differential diagnostic sophistication. Also other risk factors, such as potentially relevant lab markers (e.g. apolipoprotein E4) could not be considered. Further, with regard to the use of psychometric tools, we were predominantly limited to the use of instruments that were not specifically developed for PD patients though were robust and featured established reliability and validity. Despite this and the implementation of considerable quality assurance measures, however, we can not exclude the possibility that the administration of diagnostic tools is more variable as compared to more strictly controlled clinical-experimental designs. Yet, this potential variability is unlikely to affect the findings systematically, due to the nature of the standardized instruments as well as the representative sampling strategy, prohibiting systematic selection bias on behalf of the participating doctors. Also the current paper is the first publication of the GEPAD main study, restricting in this first paper the possibility of a more detailed presentation of the neuropsychological and neuropsychiatric findings and their implications at this point. The estimates of cognitive impairment and dementia in PD patients are partly dependent on the diagnostic measure used and are substantial with at least one third of patients affected. According to physicians' assessments, $28.6 \%$ of patients met the DSM-IV diagnostic criteria for dementia. However, neuropsychological instruments revealed considerably higher rates for clinically significant cognitive impairment of $41.8 \%$ (CDT) and 43.6\% (PANDA). Both measures, designed as simple and robust screening tools, also revealed good sensitivities (CDT: $71.1 \%$, PANDA: $73.2 \%$ ) and appropriate specificities (69.3\% and $71.4 \%)$. In contrast, the MMSE overlooks a considerable proportion of patients with clinically diagnosed dementia when the suggested cutoff score $(\leq 24)$ is used, thus revealing lower sensitivity (50 $\%$ ), questioning the utility of the MMSE for screening of dementia in PD patients.

The finding of overall at least $28 \%$ of clinically diagnosed dementia in the whole sample and considerable, up to two-fold higher rates as determined by neuropsychological instruments, is consistent with the upperbound estimates in previous studies [9, 15]. The substantially higher rates in the older patients (range 41.0-56.8 \%) additionally underscore the evidence from prospective-longitudinal studies [1] substantiating the conclusion that the risk of clinically significant dementia is considerably increased in PD versus non-PD patients.

Several factors were shown to be associated with increased rates of cognitive decline in PD. Age and particularly severity (Hoehn \& Yahr staging) of PD symptoms were associated with the clinical diagnosis of dementia and the degree of cognitive impairment in our sample [1, 33]. Cognitive impairment according to the CDT was approximately 2 times more frequent in patients in the oldest age group than in patients in the youngest age group. For the PANDA, which was administered to a subsample of patients, the frequency of cognitive impairment increased from almost one third of patients aged $\leq 65$ to more than every other patient aged $\geq$ 76 being afflicted. The frequency of dementia according to DSM-IV criteria was almost 3 times higher in patients aged $\geq 76$ than in those aged $\leq 65$ and the same held true for the rates of the MMSE.

Logistic regression analyses suggest that both age and HY stage have independent effects, both contributing to the increased prevalence of cognitive impairment. With the exception of 
the CDT, we did not find a significant effect of either gender or age of PD onset, when adjusting for age. We also could exclude the possibility that the high rates of cognitive impairment could be largely attributed to the considerable motor component of PD. One could suspect that increased rates of cognitive impairment in the neuropsychiatric instruments could largely be due to the patient's motor disturbance and his/her failure to complete complex motor-related tasks in instruments like the MMSE and CDT. Although a statistical examination of this issue revealed some indication of this, the effect size is small and did not change considerably the rates. Furthermore, if such an effect were substantial, one would expect lower rates in the PANDA, which does not contain any such complex motor tasks. This leads to the question of how high the true rates of clinically significant cognitive impairment are and what type of diagnostic measure reflects this best and with the highest sensitivity, in the presence of cognitive impairment. Accepting the differential diagnostic imprecision inherent in the study, the clinician's diagnosis according to DSM-IV can be regarded as the lower bound estimate, due to the strict diagnostic criteria, that most likely do not yet reflect the presence of PD-specific cognitive impairment in early stages. The MMSE, also frequently used as an epidemiological tool to assess cognitive impairment, in contrast reveals the lowest estimates, is largely dependent on age, and disturbingly compares poorest to the clinical diagnosis of dementia according to DSM-IV. This is in line with more general concerns, that the MMSE with its lesser accentuation of executive functions is probably not an appropriate screening tool for PD patients. In contrast the CDT yielded higher rates of cognitively impaired patients with a somewhat weaker specificity than the PANDA. The PANDA seems to be a quite sensitive instrument, particularly in younger PD patients, with a good sensitivity and specificity match as compared to clinical diagnoses in higher age groups. It might be regarded as the upper-bound estimate. In addition it is noteworthy that the scale minimizes the impact of motor disturbances on performance, as it demands only minimally motor responses (i.e. no writing or drawing necessary).

Multiple factors could have contributed to the noticeable differences in frequencies determined using the MMSE versus the CDT, PANDA and the clinical diagnosis. First, it is still under discussion if PD and PDD have the same underlying pathogenesis, that is, that cognitive abilities always deteriorate with advancing disease [21]. Thus, the relatively small proportion of advanced PD patients in our study sample could have contributed to the low frequencies of dementia. However, if this were true, the frequencies of cognitive impairment estimated with the CDT and clinical diagnosis should also have been low. Second, PD patients might perform poorly on drawing tasks as a result of their physical disabilities and not merely their cognitive deterioration. We think that this hypothesis can be excluded because tremor is not considered in the MMSE or CDT ratings. It is also possible that the MMSE is not diagnostic of the cognitive impairment typical for PD patients, as subcortical dementias (such as PDD) are understood to impact visuospatial abilities more than other abilities such as short-term memory [27]. Thus, as only one item in the MMSE refers to drawing, the total score is less sensitive to visuospatial deficits than is the CDT, which more extensively tests visuospatial ability. Visuoconstructional difficulties have been reported to be present early in the course of Lewy body disease and may also relate to fronto-subcortical dysfunction [7, 24]. Indeed, our study supports previous findings of drawing difficulties in PD patients, especially on the CDT and related gender effects [31]. Finally, comorbidity of dementia with advanced age and disease stage may contribute to the observation of visuoperceptive deficits by virtue of the presence of temporoparietal Alzheimer pathology. Idiopathic PD was also found to be associated with a substantial burden of depressive and psychotic symptoms: $21.6 \%$ of all patients in the sample exceeded the MADRS cut off for clinically significant depression and $19 \%$ had psychotic features, most frequently hallucinations. Both complications are known to be frequent complications of PD, and our estimates do not differ substantially from previous estimates [3, 30, 34]. In multiple 
regression analyses we could show, however, that depressive and psychotic symptoms reveal no substantial effects on the estimation of cognitive impairment. Although depression adds an independent statistical contribution of increased rates of cognitive impairment and dementia, the overall effect is small and would not change dramatically the rates of cognitive impairment, if the influence of depressive symptoms on measures of cognitive impairment were discarded. Furthermore, the lack of a consistent age effect for depressive and psychotic symptoms argues against the possibility that rates of cognitive impairment and dementia are largely explained by the presence of these symptoms. Instead descriptively and discarding etiopathogenetic pathways, the findings are more consistent with depression and psychotic experiences being separate phenomena adding however substantially to the patient's overall burden of disease and suffering.

Our findings are the first to provide estimates of dementia, cognitive impairment and neuropsychiatric complications based on the neurological day-to-day care sector in Germany, taken from a large representative sample of PD outpatients in office-based settings. However, our results must be interpreted with caution. The first and most important caveat is the restriction to office-based neurologists; prevalence estimates should only be interpreted with regard to this target population. Because this was not a community survey, the true prevalence of dementia in PD would necessarily be higher if more severe cases, such as hospitalized patients or nursing homes patients, were included. Likewise, PD patients in very early stages largely suffer from non-specific somatic symptoms (e.g. backache, stiffness) and thus are more likely to be under medical treatment by general practitioners than by neurologists. The latter might have resulted in overall lower estimates. Furthermore, we focused in this paper on idiopathic PD in order to describe a homogenous disease group, as results for the total sample are under review.

In conclusion, our study indicates that cognitive impairment and neuropsychiatric symptoms in PD are common in the neurological outpatient sector and occur frequently at young age, and in early stages of the disease. Screening for these complications should use sensitive neuropsychological tools for PD patients, like the PANDA, followed by a careful clinical diagnostic work up. The MMSE does not perform well in this respect, leading to a considerable underestimation of even clinically established patients with PD.

Acknowledgements The GEPAD study was supported by an unrestricted educational grant of Novartis Pharma GmbH (Nuremberg, Germany) and received support from the German Ministry of Education and Research through award number BMBF No. 01GI9901/1 (R.D.; W.H.O.). We wish to thank all cooperating physicians and patients for their participation in this study. The authors are also indebted to Nancy Chooi Ping Low, PhD, for her revision of the English. 
Fig.1 Sampling process of physidians and patents in the GEPAD study

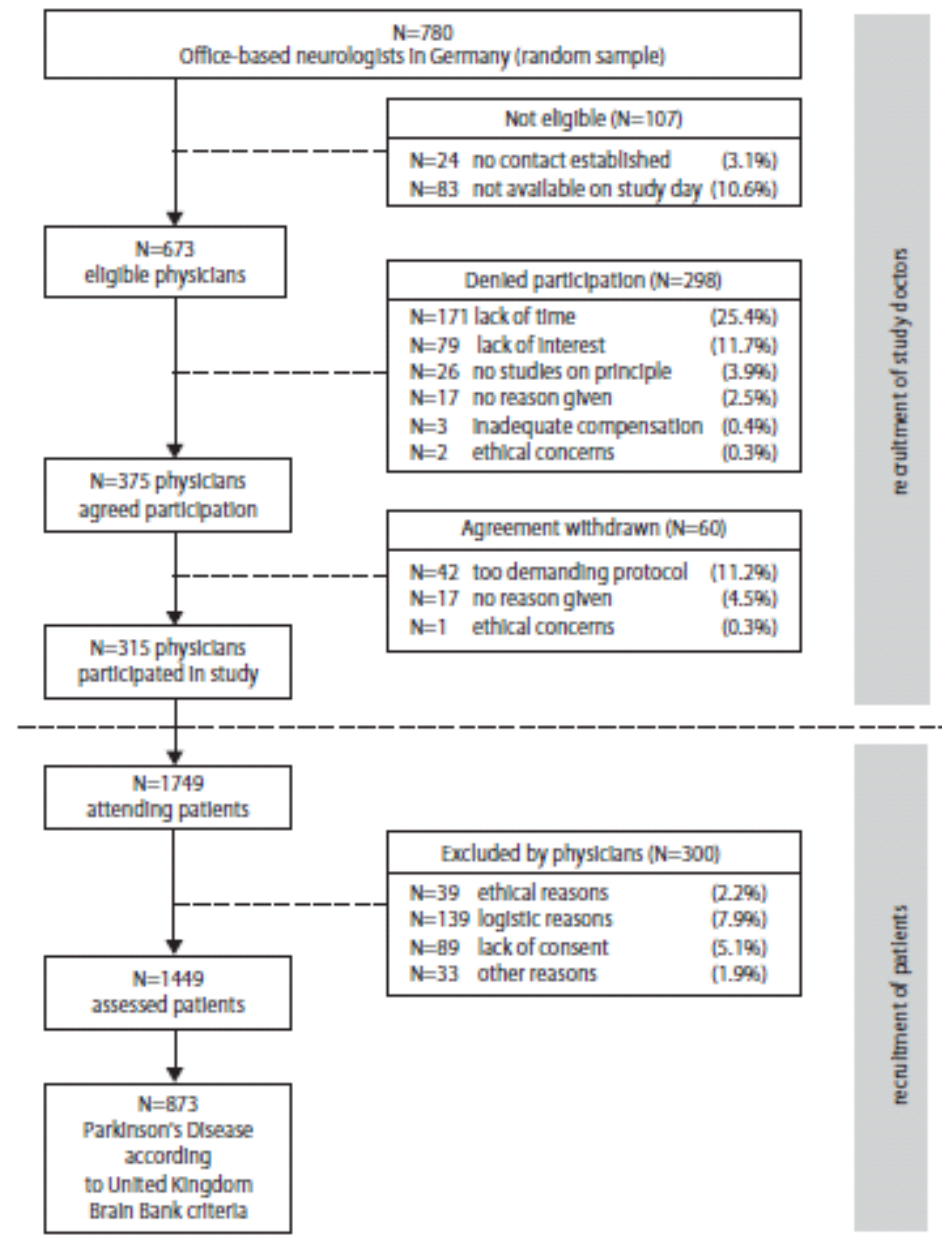

Fig.2 Frequendes of dementia (DSMN) andcognitive impairment (MMSE, CDT and PANDA) in PD patents $(\mathrm{n}=8 / 3)$
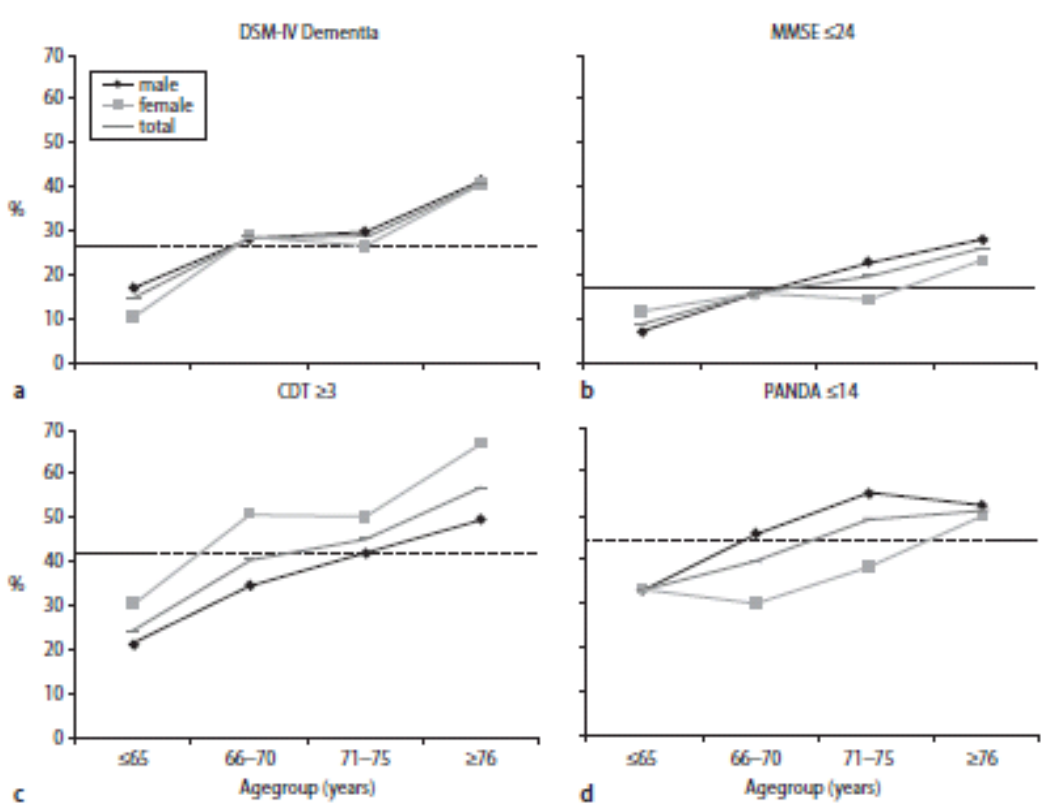
Table 1 Charactedstics of patents with Prkinson's Disease (PD)

\begin{tabular}{|c|c|}
\hline Paramater & Value* \\
\hline N & 873 \\
\hline Male, $n(\%)$ & $542(62.19)$ \\
\hline Female, n ( 9 ) & 331 (37.9 99) \\
\hline Age, years & $70.5 \pm 8.6$ \\
\hline Education, yers & $9.7 \pm 2.4$ \\
\hline Age of $P D$ onset, years & $63.8 \pm 9.9$ \\
\hline Duration of disere, yeas & $6.7 \pm 5.5$ \\
\hline Hoehn \& Yalr ${ }^{2, c}$ & $27 \pm 1.0$ \\
\hline Stage I(n, \%) & $104(1239)$ \\
\hline Stage II $(n, \%)$ & $234(27.69)$ \\
\hline Stage II (n, \%) & $3.13(40.49)$ \\
\hline Stage $N / N$ (n, 9) & 168 (19.8\%) \\
\hline UPDRSI & $28 \pm 24$ \\
\hline UPDSSIl on & $9.8 \pm 6.8$ \\
\hline UPDRS ll off & $13.2 \pm 8.6$ \\
\hline UPDESN & $3.1 \pm 3.3$ \\
\hline MMSEscore & $273 \pm 3.4$ \\
\hline PANDA score ${ }^{4}$ & $15.8 \pm 7.1$ \\
\hline CDTscore & $24 \pm 13$ \\
\hline MADRS scare & $9.4 \pm 81$ \\
\hline
\end{tabular}

- Data expressed as mean \pm SDif not otherwise indicated ${ }^{b}$ Item missing for $n=21$ subject; ' 'Stages $N$ and $V$ were pooled due to the small number of patents on stage $V(n=17) ;{ }^{4}$ administered to a subsampie of $n=289$

UPDiRS Uniffed Pakinson's Diseare Rating Scale; MMSE Mini-Mental State Exam; CDT Oodk Drawing Test; MADRS Montgomay Asberg Depression Rating Scale; PANDA Parkinson Neuropsychomatic Demertia Assessment

Table2 Assodiation of dementia (DSM-M) and cognitveimpaiment (CDT, MME, PANDA) by gender, age, and education (N = 873)

\begin{tabular}{|c|c|c|c|c|c|c|c|c|c|c|c|c|}
\hline & \multicolumn{3}{|c|}{ DSM-N aiteria for dementa } & \multicolumn{3}{|c|}{$\mathrm{CDT} \geq 3$} & \multicolumn{3}{|c|}{ MMSE $\leq 24$} & \multicolumn{3}{|c|}{ PANDA $\leq 14$} \\
\hline & $\mathrm{N}$ & $\%$ & OR' (Cl $9 \% \%)$ & $\mathrm{N}$ & $\%$ & OR' (CI96 \%) & $\mathrm{N}$ & $\%$ & OR' (Cl $95 \%)^{1}$ & $\mathrm{~N}$ & $\%$ & $O R^{\prime}(\mathrm{Cl} 96 \%)$ \\
\hline \multicolumn{13}{|l|}{ Cender } \\
\hline Male & 157 & 29.0 & reference group & 189 & 364 & reference group & 92 & 18.0 & reference group & 81 & 463 & reference group \\
\hline Female & 93 & 281 & $0.9(0.6-1.2)$ & 159 & 50.6 & $1 . T^{*}(1.3-2.3)$ & 53 & 16.7 & $0.8(0.6-1.2)$ & 6 & 39.5 & $0.7(0.4-1.1)$ \\
\hline \multicolumn{13}{|c|}{ Age group, years } \\
\hline$\leq 65$ & 33 & 14.8 & reference group & 53 & 24.4 & reference group & 19 & 8.8 & reference group & 21 & 328 & reference group \\
\hline $66-70$ & 62 & 284 & $23^{*}(1.4-3 J)$ & 86 & 40.4 & $2.1^{*}(1.4-32)$ & 33 & 15.6 & $1.9^{*}(1.1-3.5)$ & 30 & 39.5 & $13(0.7-2.7)$ \\
\hline $71-75$ & 52 & 287 & $23^{*}(1.4-3.8)$ & 76 & 45.0 & $2.5 *(1.6-3.9)$ & 33 & 19.6 & $2.5 *(1.4-4.7)$ & 30 & 492 & $20(1-41)$ \\
\hline $76 \geq$ & 103 & 41.0 & $40^{\circ}(2,6-6.3)$ & 133 & 568 & $4.1^{*}(2.7-6.1)$ & 60 & 260 & $3 . T^{*}(2.1-6.4)$ & 5 & 51.1 & $2.1^{*}(1.1-4.2)$ \\
\hline \multicolumn{13}{|c|}{ Education,yers } \\
\hline$\leq 8$ & 142 & 360 & reference group & 199 & 53.5 & reference group & 82 & 224 & reference group & 67 & 512 & reference group \\
\hline $9-10$ & 42 & 21.0 & $0.6^{*}(0.4-0.8)$ & 73 & 37.2 & $0.6^{*}(0.4-0.9)$ & 22 & 11.5 & $0.5 *(0.3-0.9)$ & $\not 2$ & 37.9 & $0.6(0.3-1.2)$ \\
\hline $11 \geq$ & 60 & 22.9 & $0.6^{*}(0.4-0.9)$ & 71 & 282 & $0.4^{*}(03-0.9)$ & 36 & 142 & $0.7(0.4-1.1)$ & 34 & 358 & $0.6(0.3-1)$ \\
\hline
\end{tabular}

1 OR Odds rato from log'stic regression, adjusted for age; CDT Oock Drawing Test; MMSE Mini-Mertal State Exam; PANDA Parkisson Neuropsychometic Dementa Assessment

Table3 Assodiation of dementia (DSM-M) and cognitveimparmert (CDT, MMS, PANDA) by PD sevelity, age of PD onset, disease duration and neuropsychiatic comobidy $(\mathrm{N}=873)$

\begin{tabular}{|c|c|c|c|c|c|c|c|c|c|c|c|c|}
\hline & \multicolumn{3}{|c|}{ DSM-N atedia for dementa } & \multicolumn{3}{|c|}{$\mathrm{Cot} \geq 3$} & \multicolumn{3}{|c|}{ MMSE $\leq 24$} & \multicolumn{3}{|c|}{ PANDA $\leq 14$} \\
\hline & $\bar{N}$ & $\%$ & OR' (CI 95\%) & $\bar{N}$ & $\%$ & OR' (CO 95\%) & $\bar{N}$ & $\%$ & ORI (CO $95 \% 9^{1}$ & $\bar{N}$ & $\%$ & $O R^{\prime}(\mathrm{C} \mid 9 \%)$ \\
\hline \multicolumn{13}{|l|}{ Diserse status } \\
\hline Hoehn \& Yahrstagel & 11 & 10.6 & reference goup & 31 & 30.4 & reference group & 8 & 7.9 & referencegoup & 7 & 180 & reference group \\
\hline Hoehn \& Yahrstagell & 48 & 20.5 & $21^{*}(1.1-43)^{\top}$ & 79 & 34.1 & $1.1(0.7-1.9)$ & 23 & 10.0 & $13(0.5-3)$ & 28 & 302 & $23(0.9-6)$ \\
\hline Hoehn \& Yahr stagelli & 105 & 30.6 & $33^{*}(1.7-64)$ & 152 & 45.9 & $1 . T^{*}(1-27)$ & 63 & 19.0 & $24^{\circ}(1.1-5.3)$ & 57 & 50.9 & $4.5^{*}(1.8-11.1)$ \\
\hline Hoehn \& Yahr stageN N & 81 & 48.2 & $63^{*}(3.1-128)$ & $\pi 7$ & 53.1 & $2 \sigma^{\circ}(1.2-3.5)$ & 47 & 33.1 & $4 T^{*}(2.1-10.5)$ & 31 & 620 & $6.8^{\circ}(2.5-18.5)$ \\
\hline Age of onset $<59$ years & 48 & 21.0 & referencegroup & 66 & 29.6 & reference group & 22 & 10.1 & referencegoup & 32 & 478 & reference group \\
\hline Age of onset $\geq 59$ yeas & 180 & 30.7 & $0.7(0.5-12)$ & 255 & 45.7 & $1.0(0.6-1.5)$ & 109 & 19.5 & $1.1(0.6-2)$ & 84 & 412 & $0.3^{*}(0.1-0.8)$ \\
\hline Dsease duration $\leq 5$ years & 104 & 253 & referencegoup & 150 & 38.0 & reference group & 59 & 14.9 & reference goup & 56 & 376 & reference group \\
\hline Dsease duration $\geq 6$ years & 124 & 30.6 & $13(0.9-1.8)$ & 171 & 443 & $13(1-1.7)$ & 72 & 189 & $13(0.9-1.9)$ & 60 & 492 & $1.6(1-2.5)$ \\
\hline \multicolumn{13}{|l|}{ Psychotic symptoms ${ }^{2}$} \\
\hline isual misperceptions & 69 & 548 & $3.8^{*}(2.5-5.7)$ & 67 & 583 & $2.1^{*}(1.4-3.2)$ & 39 & 35.1 & $3.1^{*}(1.9-4.8)$ & 24 & 57.1 & $1.9(1-3.7)$ \\
\hline halluchations & 70 & 603 & $4.8^{*}(3.1-7.3)$ & 68 & 65.4 & $3.0(2-46)$ & 37 & 363 & $3.1 *(2-5)$ & 21 & 63.6 & $25^{*}(1.2-53)$ \\
\hline parandid symptoms & 26 & 722 & $69^{*}(3.1-15.3)$ & 22 & 68.8 & $3.1 *(1.4-6.9)$ & 13 & 40.6 & $33 *(1.6-68)$ & 7 & 63.6 & $22(0.6-7.5)$ \\
\hline delusions & 14 & 63.6 & $45^{*}(1.8-11.4)$ & 16 & 80.0 & $622^{*}(2-19.6)$ & 9 & 45.0 & $4.1^{*}(1.6-10.8)$ & 5 & 62.5 & $23(0.5-10.6)$ \\
\hline any & 91 & 548 & $41^{*}(28-6)$ & 87 & 58.4 & $22^{*}(1.6-3.2)$ & 49 & 333 & $3.0(2-15)$ & $\not z$ & 563 & $1.8(1-3.3)$ \\
\hline \multicolumn{13}{|l|}{ MADRS scare } \\
\hline$\leq 13$ & 132 & 21.6 & reference group & 223 & 372 & rferencegroup & 85 & 143 & referencegroup & 84 & 40.8 & reference group \\
\hline$\geq 14$ & 109 & 50.2 & $3 . T^{*}(26-5.2)$ & 113 & 57.4 & $23^{*}(1.6-3.2)$ & 55 & 28.2 & $23^{*}(1.6-3.4)$ & 36 & 507 & $1.5(0.9-26)$ \\
\hline
\end{tabular}

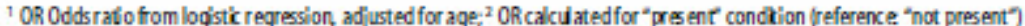

CDT Oodk Drawing Test, MME Mini-Mental State Exam, PAVDA Parkiss on Neuropsychomatic Dementia Assessment MADPS Mont gomary Asberg Depression RatingScal e 


\section{References}

1. Aarsland D, Andersen K, Larsen JP, Lolk A, Kragh-Soerensen P (2003) Prevalence and Characteristics of Dementia in Parkinson's Disease - An 8-Year Prospective Study. Arch Neurol 60:387-392

2. Aarsland D, Andersen K, Larsen JP, Lolk A, Nielsen H, Kragh-Soerensen P (2001) Risk of dementia in Parkinson's disease: A community-based, prospective study. Neurology 56:730-736 3. Aarsland D, Larsen JP, Lim NG, Janvin C, Karlsen K, Tandberg E, Cummings J (1999) Range of neuropsychiatric disturbances in patients with Parkinson's disease. J Neurol Neurosurg Ps 67:492-496 4. Adler C (2005) Nonmotor Complications in Parkinson's Disease. Movement Disord 20:S23-S29 5. American Psychiatric Association (2000) Diagnostic and Statistical Manual of Mental Disorders Text revision. American Psychiatric Association, Washington, DC

6. Campenhausen S, Bornschein B, Wick R, Bötzel K, Sampaio C, Poewe W, Oleson J, Oertel W, Siebert U, Berger K, Dodel R (2005) Prevalence and incidence of Parkinson's disease in Europe. Eur Neuropsychopharm 15:473-490

7. Cormack F, Aarsland D, Ballard C, Tovee M (2004) Pentagon drawing and neuropsychological performance in Dementia with Lewy bodies, Alzheimer's disease, Parkinson's disease and Parkinson's disease with dementia. Int J Geriatr Psych 19:371-377

8. Efron B, Tibshirani R (1993) An introduction to the bootstrap. Chapman and Hall, London 9. Emre M (2003) What causes mental dysfunction in Parkinson's disease? Movement Disord 18:6371

10. Fahn S (1987) Unified Parkinson's Disease Rating Scale. In: Fahn S, Calne D (eds) Recent Developments in Parkinson's Disease. MacMillan Healthcare Information, Florham Park, NJ, pp 153163

11. Folstein M, Folstein S, McHugh P (1975) Mini-Mental state: a practical method for grading the mental state of patients by the clinician. J Psychiatr Res 12:189-198

12. Hobson P, Meara J (2004) Risk and incidence of dementia in a cohort of older subjects with Parkinson's disease in the United Kingdom. Movement Disord 19:1043-1049

13. Hoehn M, Yahr M (1967) Parkinsonism: onset, progression and mortality. Neurology 17:427-442 14. Hughes A, Daniel S, Kilford L, Lees A (1992) Accuracy of clinical diagnosis of idiopathic Parkinson's disease: a clinicopathological study of 100 cases. J Neurol Neurosurg Ps 55:181-184 15. Hughes T, Ross H, Musa S, Bhattacherjee S, Nathan R, Mindham R, Spokes E (2000) A 10-yearstudy of the incidence of and factors predicting dementia in Parkinson's disease. Neurology 54:1596-1603

16. Huse D, Schulman K, Orsini L, Castelli-Haley J, Kennedy S, Lenhart G (2005) Burden of illness in Parkinson's disease. Movement Disord 20:1449-1454

17. Kalbe E, Calabrese P, Kohn N, Hilker R, Riedel O, Wittchen HU, Dodel R, Otto J, Ebersbach G, Kessler J (2007) Screening for cognitive deficits in Parkinson's Disease with the Neuropsychometric Dementia Assessment (PANDA) instrument. Parkinsonism Relat D (in press)

18. Kalbe E, Riedel O, Kohn N, Dodel R, Calabrese P, Kessler J (2007) Sensitivität und Spezifität des "Parkinson Neuropsychometric Dementia Assessment" (PANDA): Ergebnisse der GEPAD-Studie. Aktuel Neurol 34:140-146

19. Klockgether T (2004) Parkinson's disease: clinical aspects. Cell Tissue Res 318:115-120

20. Leentjens A, Verhey F, Lousberg R, Spitsbergen H, Wilmink F (2000) The validity of the Hamilton and Montgomery-Asberg Depression Rating Scales as screening and diagnostic tools for depression in Parkinson's disease. Int J Geriatr Psych 15:644-649

21. Louis E, Schupf N, Manly J, Marder K, Tang M-X, Mayeux R (2005) Association between mild parkinsonian signs in mild cognitive impairment in a community. Neurology 64:1157-1161

22. Miyasaki J, Shannon K, Voon V, Ravina B, Kleiner-Fisman G, Anderson K, Shulman L, Gronseth G, Weiner WJ (2006) Practice Parameter: Evaluation and treatment of depression, psychosis, and dementia in Parkinson disease (an evidence-based review). Neurology 66:996-1002

23. Montgomery S, Asberg M (1979) A new depression scale, designed to be sensitive to change. Brit J Psychiat 134:382-389

24. Muslimovic D, Post B, Speelman J, Schmand B (2005) Cognitive profile of patients with newly diagnosed Parkinson disease. Neurology 65:1239-1245 
25. National Institute of Mental Health (1976) CGI: Clinical Global Impressions. National Institute of Mental Health, Chevy Chase, MD

26. Nishiwaki Y, Breeze E, Smeeth L, Bulpitt C, Peters R, Fletcher A (2004) Validity of the ClockDrawing-Test as a screening tool for cognitive impairment in the elderly. Am J Epidemiol 160:797807

27. Noe E, Marder K, Bell K, Jacobs D, Manly J, Stern Y (2004) Comparison of dementia with Lewy bodies to Alzheimer's disease and Parkinson's disease with dementia. Movement Disord 19:60-67 28. Riedel O, Dodel R, Spottke A, Deuschl G, Förstl H, Henn F, Heuser I, Oertel W, Reichmann H, Riederer P, Trenkwalder C, Wittchen H-U (2006) Wie beurteilen Ärzte die Häufigkeit demenzieller, depressiver und psychotischer Symptome bei Patienten mit der Parkinson-Krankheit? Eine Befragung von 500 Fachärzten im ambulanten Versorgungssektor Deutschlands. Aktuel Neurol 33:374-380 29. Royall RM (1986) Model robust confidence intervals using maximum likelihood estimators. Int Stat Rev 54:221-226

30. Schrag A, Jahanshahi M, Quinn N (2001) What contributes to depression in Parkinson's disease? Psychol Med 31:65-73

31. Seigerschmidt E, Mösch E, Siemen M, Förstl H, Bickel H (2002) The clock drawing test and questionable dementia: reliability and validity. Int J Geriatr Ps 17:1048-1054

32. Shulman K (2000) Clock-drawing: Is it the ideal cognitive screening test? Int J Geriatr Ps 15:548561

33. Vingerhoets G, Verleden S, Santens P, Miatton M, De Reuck J (2003) Predictors of cognitive impairment in advanced Parkinson's disease. J Neurol Neurosurg Ps 74:793-796

34. Wichowicz H, Slawek J, Derejko M, Cubala W (2006) Factors associated with depression in Parkinson's disease: a cross-sectional study in a Polish population. Eur Psychiat 21:516-520 